\title{
A novel system to monitor mitochondrial translation in yeast
}

\author{
Tamara Suhm ${ }^{1}$, Lukas Habernig ${ }^{2}$, Magdalena Rzepka ${ }^{1}$, Jayasankar Mohanakrishnan Kaimal ${ }^{3}$, Claes $^{2}$ \\ Andréasson ${ }^{3}$, Sabrina Büttner ${ }^{2,3}$ and Martin Ott ${ }^{1, *}$ \\ ${ }^{1}$ Department of Biochemistry and Biophysics, Stockholm University, SE-10691 Stockholm, Sweden. \\ ${ }^{2}$ Institute of Molecular Biosciences, University of Graz, A-8010 Graz, Austria. \\ ${ }^{3}$ Department of Molecular Biosciences, the Wenner-Gren Institute, Stockholm University, SE-10691 Stockholm, Sweden. \\ * Corresponding Author: \\ Martin Ott, Department of Biochemistry and Biophysics, Stockholm University, SE-10691 Stockholm, Sweden; E-mail: \\ martin.ott@dbb.su.se
}

\begin{abstract}
The mitochondrial genome is responsible for the production of a handful of polypeptides that are core subunits of the membrane-bound oxidative phosphorylation system. Until now the mechanistic studies of mitochondrial protein synthesis inside cells have been conducted with inhibition of cytoplasmic protein synthesis to reduce the background of nuclear gene expression with the undesired consequence of major disturbances of cellular signaling cascades. Here we have generated a system that allows direct monitoring of mitochondrial translation in unperturbed cells. A recoded gene for superfolder GFP was inserted into the yeast (Saccharomyces cerevisiae) mitochondrial genome and enabled the detection of translation through fluorescence microscopy and flow cytometry in functional mitochondria. This novel tool allows the investigation of the function and regulation of mitochondrial translation during stress signaling, aging and mitochondrial biogenesis.
\end{abstract}

doi: $10.15698 /$ mic2018.03.621

Received originally: 13.10.2017;

in revised form: 22.12.2017,

Accepted 02.01.2018,

Published 13.01.2018.

Keywords: mitochondrial translation, flow cytometry, superfolder GFP, strain engineering.

\section{Abbreviations:}

CAP-chloramphenicol, OXPHOS-oxidative phosphorylation, sfGFP - superfolder GFP,

UTR - untranslated region.

\section{INTRODUCTION}

In Saccharomyces cerevisiae the mitochondrial genome encodes eight proteins of which seven are membrane proteins and core subunits of the oxidative phosphorylation system (OXPHOS) [1]. Respiratory activity, therefore, depends on mitochondrial translation, but the molecular mechanisms, regulation and timing of mitochondrial translation are largely unexplored [2]. A major obstacle when analyzing mitochondrial translation in cells is the need to inhibit cytosolic translation in order to follow incorporation of radiolabeled amino acids into mitochondrial translation products [3]. Such pharmacological inhibition impacts severely on cellular homeostasis and alters signaling cascades. For example, inhibition of cytosolic translation using cycloheximide leads to an increase in free amino acid levels, which in turn activates TOR signaling [4-6] and thus affects cell growth, nutrient signaling and life span [7-9]. Additionally, cycloheximide treatment alters protein degradation [10] and acutely impacts on mitochondrial translation within very short time frames [11]. To directly monitor mito- chondrial protein synthesis without interfering with cellular protein homeostasis new experimental tools are needed.

Employing biolistic transformation we have integrated a gene encoding superfolder GFP into the mitochondrial genome. This reporter is compatible with mitochondrial respiratory function and enables the direct detection of mitochondrial translation in vivo as GFP fluorescence. This novel tool will facilitate future studies on the regulation and timing of mitochondrial translation.

\section{RESULTS AND DISCUSSION}

Integration of a superfolder GFP gene into the mitochondrial genome

In a pioneering previous study, a gene encoding fluorescence-enhanced GFP was inserted into the mitochondrial genome to replace the open reading frame of COX3 [12]. The resulting strain was respiration deficient and expressed only weak fluorescence, which limited its usefulness in fluorescence microscopic and flow cytometric experiments. This limitation could be explained by poor folding of GFP expressed in the context of the mitochondrial 
A

Biolistic transfromation of DFS160 rho ${ }^{\circ}$ with pPT24*-sfGFP ${ }^{m}$

Cytoduction of this strain with cox2-62 to insert $\operatorname{cox} 2:: s f G F P^{m}$ and to restore respiration

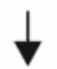

Transfer of cox2::sfGFP ${ }^{m}$ mtDNA to W303
B

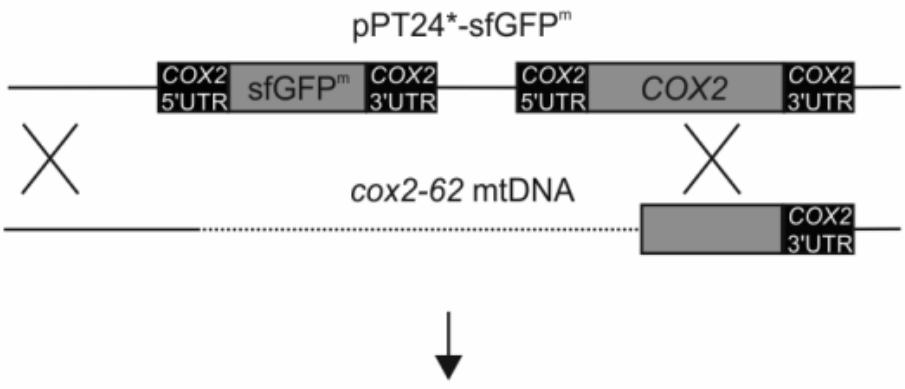

C
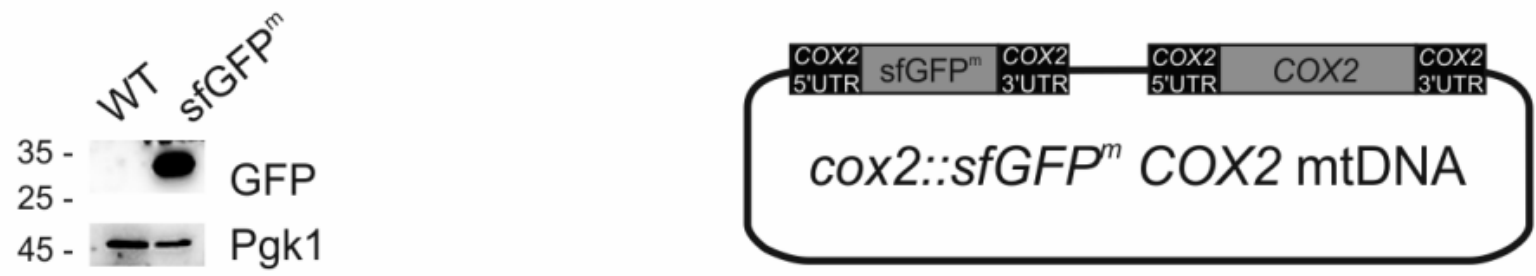

FIGURE 1: Integration of $s f G F P^{m}$ into the mitochondrial genome. (A) Strain construction strategy. (B) Schematic representation of integration of the reporter into the mitochondrial genome via homologous recombination. 5' and $3^{\prime}$ UTR of COX2 drive the expression of sfGFP ${ }^{m}$. (C) Control of successful expression of sfGFP ${ }^{m}$ via Western blotting using a GFP antibody. 3-phosphoglycerate kinase (Pgk1) served as a loading control.

translation system [13], which is specialized on the production of membrane proteins. To circumvent the folding problem, we employed superfolder GFP (sfGFP), which folds with enhanced kinetics resulting in a more stable protein [14]. To allow translation of the mRNA by mitochondrial ribosomes its coding sequence was flanked by the authentic 5' and 3' untranslated regions (UTR) of COX2 [15-18], coding for the cytochrome oxidase subunit Cox2. To avoid the respiratory deficiency associated with inactivation of mitochondrial genes, we engineered a new mitochondrial genome that coded for sfGFP as an additional ninth open reading frame. The final engineered gene was termed $s f G F P^{m}$ and was cloned into the pPT24* plasmid (Fig 1A-B), yielding a plasmid (pPT24*-sfGFP ${ }^{m}$ ) that contained COX2 5'UTR-sfGFP ${ }^{m}$-COX2 $3^{\prime}$ UTR as well as the authentic COX2, including its upstream sequences (Fig 1B). pPT24*-sfGFP ${ }^{m}$ was delivered into a strain that lacks mtDNA $\left(r h o^{\circ}\right)$ using biolistic transformation [19]. After selection, the resulting strain that carried pPT24*-sfGFP ${ }^{m}$ was used to cytoduce strain cox2-62, which lacks a functional COX2 gene, to gain respiratory competence by homologous recombination of the two mitochondrial genomes [20]. Finally, we transferred the novel mitochondrial genome into the strain $\mathrm{W} 303$ resulting in the strain sfGFP ${ }^{\mathrm{m}}$. Western blot analysis demonstrated that this engineered genome expressed readily detectable levels of sfGFP $^{\mathrm{m}}$ (Fig 1C).

\section{Expression of mitochondrially encoded $s f G F P^{m}$ does not disturb mitochondrial function}

We assessed the impact of $s f G F P^{m}$ expression on mitochondrial function by monitoring respiratory growth on non-fermentable medium and found that it was similar between wild type and the sfGFP ${ }^{m}$ strain (Fig 2A and 2B). As predicted, steady state levels of respiratory chain subu- nits were unchanged and showed the expected increase when cells were grown in non-fermentable compared to fermentable medium (Fig 2C). Finally, we checked for respiratory chain assembly into supercomplexes as well as cytochrome oxidase activity and found that both were unchanged compared to wild type (Fig 2D). We therefore conclude that expression of $\mathrm{sfGFP}^{\mathrm{m}}$ does not alter the assembly of respiratory complexes or the general respiratory competence of the cells.

\section{Expression of sfGFP ${ }^{m}$ followed by flow cytometry}

We next explored the possibility to use sfGFP ${ }^{m}$ as an optical readout for mitochondrial translation. First we checked the steady-state levels of sfGFP ${ }^{m}$ by Western blotting during respiration and fermentation to verify that mitochondrially encoded sfGFP follows the same expression pattern as other mitochondrially encoded proteins. As expected sfGFP $^{m}$ protein levels increased in the presence of galactose and glycerol after 6 and 8 hours in the same way as Cox2 levels (Fig 3A-B). Next we determined GFP fluorescence by flow cytometry under the same conditions and observed an increase in the fluorescent signal when cells were grown for 6,8 and 10 hours in galactose or glycerol containing medium (Fig 3C-D). To control for mitochondrial biogenesis we used mCherry-tagged Cit1, a nuclear encoded protein that is imported into mitochondria from the cytosol [21]. Similar to sfGFP ${ }^{m}$, Cit1-mCherry levels also increased when grown in the presence of galactose or glycerol, indicating a general increase in mitochondrial biogenesis induced by galactose and glycerol (Fig 3C-D). In sum, these results confirm that sfGFP ${ }^{m}$ represents a functional reporter for the analysis of mitochondrial translation in vivo without the need to inhibit cytosolic translation. Flow cytometry as quantitative readout opens up many 
A
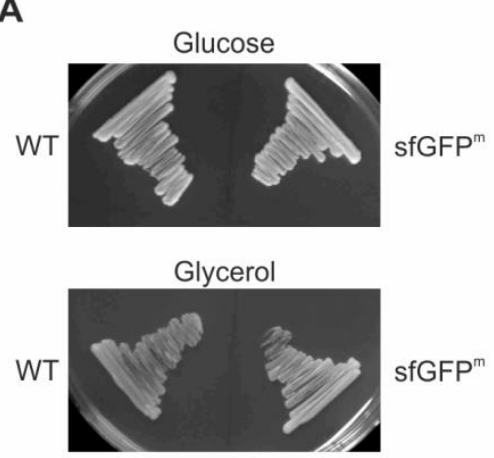

B

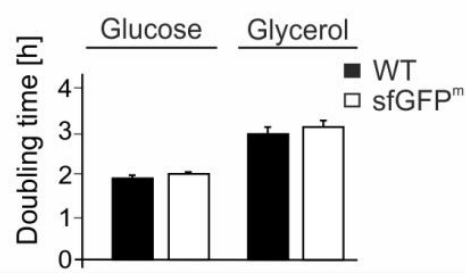

C
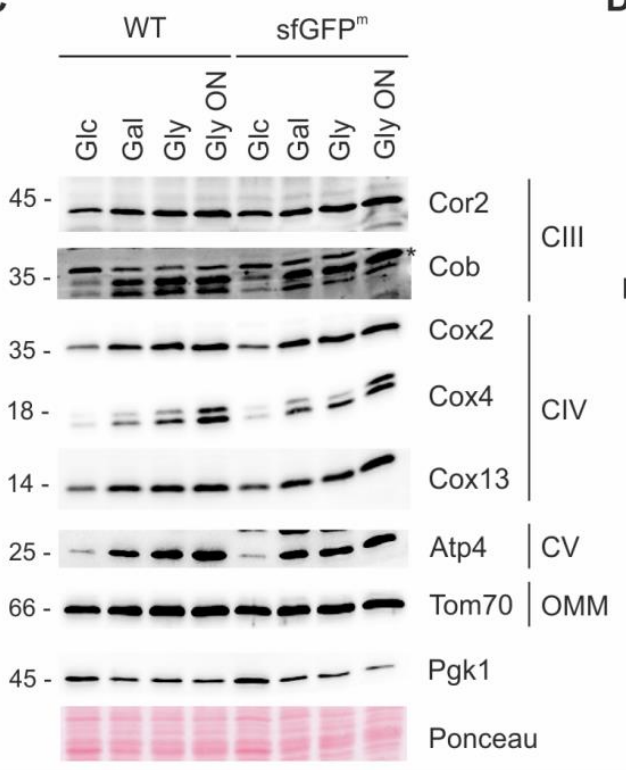

D

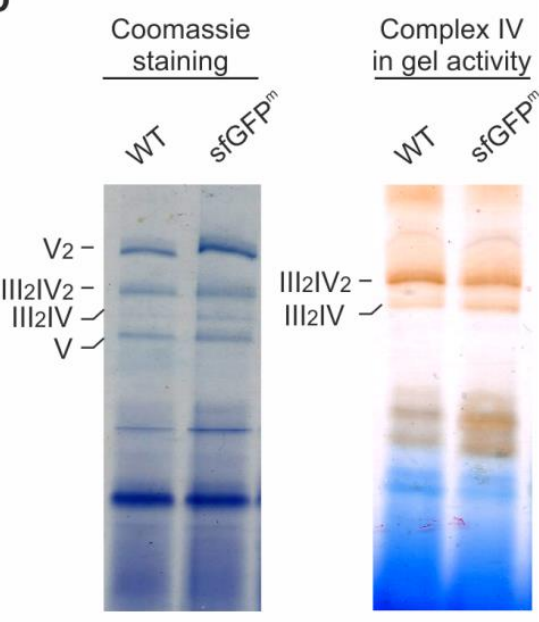

FIGURE 2: Expression of $\boldsymbol{s f G F P}^{m}$ does not disturb mitochondrial function. (A) Wild type (WT) and sfGFP ${ }^{\mathrm{m}}$ were streaked out on fermentable (Glucose) and non-fermentable (Glycerol) medium. (B) Doubling time during exponential growth phase in glucose (Glc) and glycerol (Gly). Data represent the mean of three independent experiments +/- SD. (C) Steady state levels of OXPHOS subunits in WT and sfGFP ${ }^{m}$ during exponential phase in glucose (Glc), galactose (Gal) or glycerol (Gly). Whole cell extracts were separated on SDS-PAGE and analyzed with Western Blotting using the indicated antibodies. (D) Isolated mitochondria of WT and sfGFP ${ }^{\mathrm{m}}$ were lysed in digitonin and protein complexes were separated by blue-native PAGE. Supercomplexes (III2IV and $\mathrm{III}_{2} \mathrm{IV}_{2}$ ) were either visualized by Coomassie staining or complex IV in-gel activity assay.

possibilities to interrogate mitochondrial translation with single cell resolution and allows the combination of sfGFP $^{m}$ with additional fluorophores for a multitude of simultaneous analyses.

\section{sfGFP $^{m}$ allows detection of changes in mitochondrial gene expression}

We determined the stability of sfGFP $^{\mathrm{m}}$ by inhibiting mitochondrial translation using chloramphenicol (CAP). Using immunoblotting coupled with detection by near-infrared fluorescence, we found that sfGFP ${ }^{m}$ levels declined over four hours after treatment with CAP (Fig 4A). Accordingly, flow cytometry confirmed that already two hours after block of mitochondrial translation via CAP, a decrease in GFP intensity was detectable. While this loss of sfGFP $^{m}$ signal became even more evident over time (Fig 4B), the nuclear encoded mitochondrial protein Cit1-mCherry was stable (Fig 4B). To further test the reporter construct, we made use of glucose repression of mitochondrial function [22-24]. When adding glucose to non-fermentable medium, mitochondrial biogenesis as well as translation and OXPHOS assembly are repressed. During glucose repression, we observed a pronounced decline in sfGFP $^{\mathrm{m}}$ levels by Western blotting and flow cytometric evaluation. As expected, Cit1-mCherry levels were also decreased by glucose-induced repression of mitochondrial biogenesis (Fig 4C-D). Taken together, both western blot analysis and flow cytometric evaluation of $\mathrm{sGFP}^{\mathrm{m}}$ faithfully report the specific inhibition of mitochondrial translation by CAP as well as the general down regulation of mitochondrial biogenesis during glucose repression.

\section{sfGFP $^{m}$ visualized by fluorescence microscopy}

Finally, we visualized sfGFP ${ }^{m}$ via fluorescence microscopy. Cells grown in non-fermentable medium in exponential phase showed mitochondrially localized GFP fluorescence (Fig 5), as evidenced by co-localization with Cit1-mCherry (Supplemental movie). This opens the possibility to employ sfGFP $^{m}$ not only as a reporter for mitochondrial translation but also to follow mitochondrial movement during cell division, mitophagy, or mitochondrial biogenesis.

In conclusion, we have established a functionally neutral mitochondrially encoded GFP reporter, termed sfGFP ${ }^{m}$, which is easily monitored via Western blotting, flow cytometry and microscopy. Our reporter allows studying mitochondrial translation in vivo without poising cytoplasmic translation and therefore enables detailed studies of mitochondrial function without perturbing cell physiology. The versatile nature of the new mitochondrial genome described here opens up new venues for the investigation of mitochondrial gene expression by optical methods in respiring cells.

\section{MATERIALS AND METHODS Generation of $s f G F P^{m}$}

A gene encoding superfolder GFP (sfGFP) was was recoded to match mitochondrial codon usage, synthesized (Invitrogen, Life technologies, GeneArt) and cloned into the pKM vector using Ndel and Xhol. pKM contains the 5' and 3'UTR of COX2 


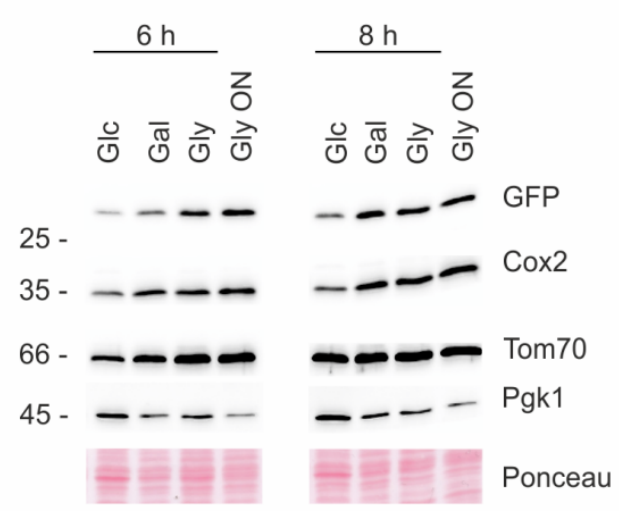

B

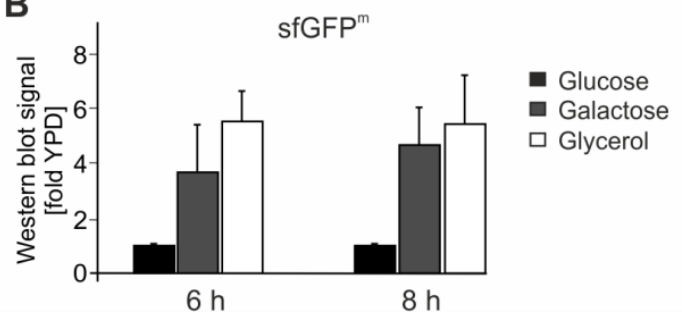

C
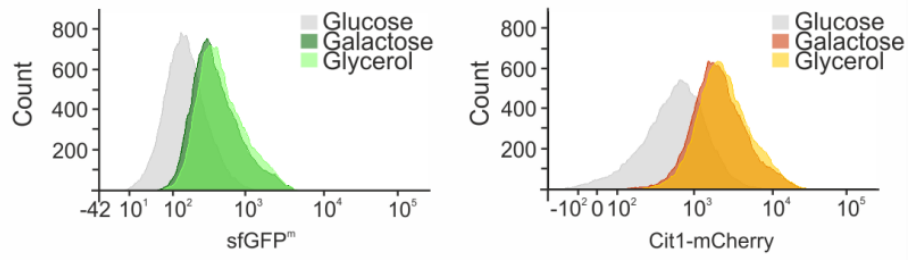

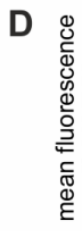
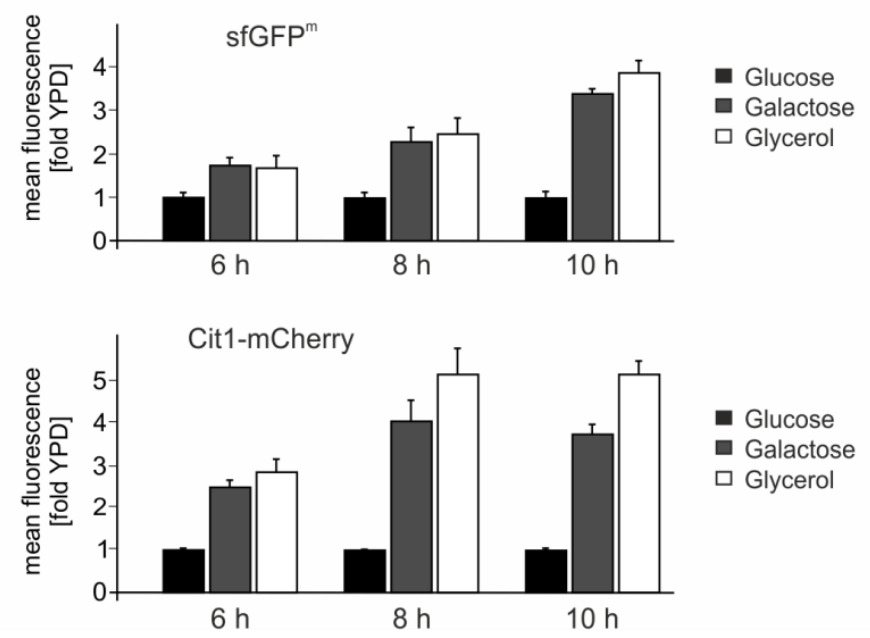

FIGURE 3: Analysis of sfGFP ${ }^{m}$ expression. (A) Cells from an overnight culture in YPD were grown to exponential phase for six or eight hours on different carbon sources. Whole cell extracts were separated on SDS-PAGE and analyzed with Western Blotting using the indicated antibodies. (B) Quantification of Western blotting signal of three independent experiments represented as means +/- SD. GFP signals were normalized to that of 3-phosphoglycerate kinase (Pgk1) and the YPD signal was set to 1. (C) Flow cytometry histogram showing sfGFP ${ }^{\mathrm{m}}$ and Cit1mCherry signals of cells grown on the indicated carbon sources. (D) As in A) but cells were analyzed using flow cytometry. Signal intensities of sfGFP ${ }^{\mathrm{m}}$ and Cit1-mCherry were quantified from eight independent samples, and the fluorescence intensity recorded for a strain lacking any fluorescent tag was subtracted as background. Signals on galactose or glycerol were normalized to the respective time point on glucose and the mean +/- SEM is depicted.

between two EcoRI restriction sites. sfGFP ${ }^{m}$ flanked by the $5^{\prime}$ and $3^{\prime}$ UTR of COX2 was then re-cloned from pKM into PPT24* using the EcoRI restriction sites $[15,25,26]$. pPT24*-sfGFP ${ }^{m}$ contains a part of authentic mtDNA, the sfGFP ${ }^{\mathrm{m}}$ under control of the $5^{\prime}$ and $3^{\prime}$ UTR of COX2 and, downstream of sfGFP ${ }^{m}$, the full COX2 gene including its $5^{\prime}$ and $3^{\prime}$ UTR. pPT24*-sfGFP ${ }^{m}$ was integrated into the mitochondrial genome via biolistic transformation and homologous recombination. Briefly, the rho ${ }^{0}$ kar1-1 DFS160 strain [27] was grown in YP medium containing $2 \%$ raffinose and spread on SD-Leu plates containing sorbitol (1 M). The nuclear marker plasmid pRS315 $(5 \mu \mathrm{g})$, as well as the pPT24*-sfGFP ${ }^{m}(10-15 \mu \mathrm{g})$, were precipitated on tungsten particles $\left(100 \mu \mathrm{l}\right.$ tungsten particle $(0.5 \mu \mathrm{m}), 1 \mathrm{M} \mathrm{CaCl}_{2}, 16 \mathrm{mM}$ spermidine) and spread on flying discs. The particles were bombarded onto a lawn of yeast cells with a particle gun (Biorad, USA). After four to five days positive nuclear transformants were collected on a master plate and crossed to a cox2-62 tester strain $[20,28]$. The diploids were screened for respiratory growth. This crossing step was repeated twice with positive clones. Eventually the stable synthetic rho strain containing pPT24*-sfGFP ${ }^{m}$ was repopulated with mtDNA via cytoduction with a strain containing the cox2-62 mutation. A haploid clone that regained respiratory competence was identified and used to transfer mitochondrial DNA into W303 rho ${ }^{\circ}$ to create strain MOY1355 (Mat $\boldsymbol{\alpha}$ ade2-1 his3-11,15 trp1-1 leu2-3,112 ura3-1, sfGFPm::cox2, COX2). An mCherry-tagged variant of Cit1 was generated by replacing the stop codon of the endogenous open reading frame with a sequence encoding mCherry followed by a TRP1 selection cassette to yield strain MOY1360 (Mat $\boldsymbol{\alpha}$ ade2-1 his3-11,15 trp1-1 leu2-3,112 ura3-1, CIT1-mCherry::TRP1, sfGFPm::cox2, COX2). Cells were grown at $30^{\circ} \mathrm{C}$ in $1 \%$ yeast extract, $2 \%$ peptone medium supplemented with $2 \%$ dextrose (YPD), $2 \%$ galactose (YPGal) or $2 \%$ glycerol (YPG) under shaking. When indicated $2 \mathrm{mg} / \mathrm{mL}$ chloramphenicol (dissolved in ethanol) or $150 \mathrm{mg} / \mathrm{mL}$ cycloheximide (dissolved in water) were added.

\section{SDS-PAGE and Western Blotting}

Proteins were extracted by alkaline lysis with $370 \mathrm{mM} \mathrm{NaOH}$, precipitated with $8.33 \%$ TCA and pellets were resuspended in reducing sample buffer (50 $\mathrm{mM}$ Tris- $\mathrm{HCl}$ pH 6.8, 2\% SDS, 10\% glycerol, $100 \mathrm{mM}$ DTT) (modified from [29]). Proteins were separated using $16 \%$ acrylamide, $0.2 \%$ bisacrylamide SDSPAGE and blotted on nitrocellulose membrane. Western blot signals were quantified using ImageJ. For immunoblot analyses upon treatment with chloramphenicol (CAP) or addition of $2 \%$ glucose, cells equivalent of 4 OD600 were harvested at indicated time points. Proteins were extracted by alkaline lysis with $1.8 \mathrm{M} \mathrm{NaOH}, 7.5 \% \beta$-mercaptoethanol and precipitated with $27.5 \%$ TCA. Pellets were resuspended in $100 \mu \mathrm{L}$ urea loading buffer (200 mM Tris-HCl pH 6.8, 8 M Urea, 5\% SDS, 1 mM EDTA, 0.02\% Bromphenolblue, $15 \mathrm{mM}$ DTT) and incubated at $65^{\circ} \mathrm{C}$ for $10 \mathrm{~min}$. Proteins were separated on $12.5 \%$ Tris- 
A

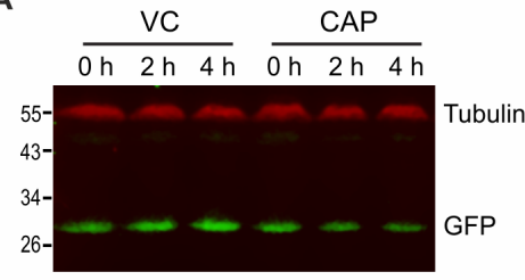

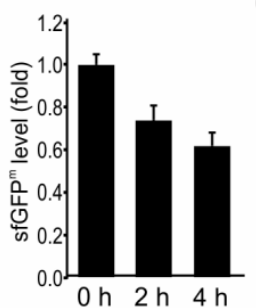

C
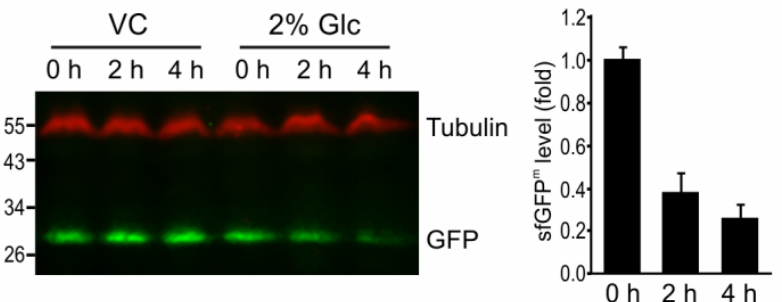

B
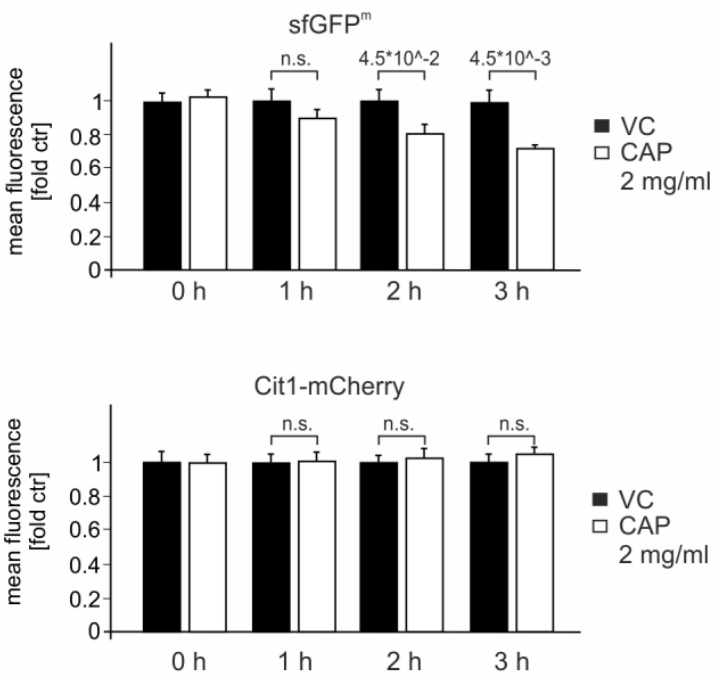

D

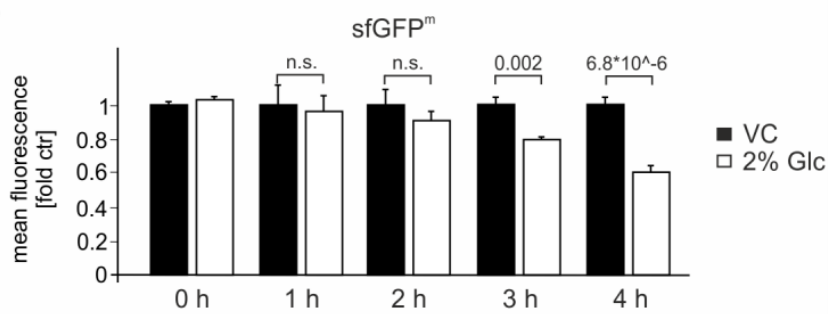

Cit1-mCherry

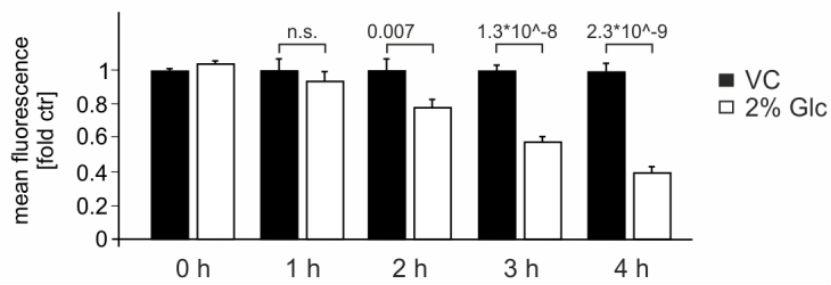

FIGURE 4: Impact on sfGFP ${ }^{m}$ protein levels by inhibition of mitochondrial translation or glucose repression. (A) Cells from an overnight culture in YPD were grown to exponential phase for six hours in glycerol. Cells were treated with $2 \mathrm{mg} / \mathrm{ml}$ chloramphenicol (CAP) or vehicle control (VC) for the indicated times and whole cell extracts were separated on SDS-PAGE and analyzed with Western Blotting using the indicated antibodies. The fluorescence signals of 6 independent experiments were quantified and the GFP signals were normalized to the tubulin signals. Data is depicted as fold of the respective untreated time point and the mean +/- SEM is displayed. (B) As in (A) but cells were analyzed using flow cytometry. Signals of sfGFP ${ }^{m}$ and Cit1-mCherry were quantified from eight independent samples, and the fluorescence intensity recorded for a strain lacking any fluorescent tag was subtracted as background before signals from treated cells were normalized to control values. Numbers indicate significance values from student t-test. (C) Cells were grown as in A, but exposed to glucose. (D) As in (B) but cells were exposed to glucose. Numbers indicate significance values from student t-test.

glycine SDS-PAGE and transferred onto nitrocellulose membranes. Membranes were blocked for 1 hour with $1 \%$ skim milk in TBST (0.05\% Tween) and incubated over night with antibodies directed against alpha-tubulin (ab184970) and GFP (Roche 11814460001$)$ in TBST (0.2\% Tween + $1 \%$ skim milk). Membranes were washed four times with TBST (0.05\% Tween) and incubated for 1 hour with the following near-infrared fluorescent secondary antibodies: anti-mouse IRDye $800 \mathrm{CW}$ 926-32210 and anti-rabbit IRDye 680RD 926-68071 diluted $1: 20000$ in TBST $(0.2 \%$ Tween $+0.01 \%$ SDS). Membranes were washed four times with TBST $(0.05 \%$ Tween), rinsed twice with TBS and signals were analyzed using Odyssey Fc (Li-499 Cor Biosciences, Lincoln, NE) and Image Studio Lite software.

\section{Native-PAGE and complex IV activity}

For native-PAGE, isolated mitochondria $(100 \mu \mathrm{g})$ were solubilized (50 mM BisTris, $25 \mathrm{mM} \mathrm{KCl}, 1 \mathrm{mM}$ EDTA, $2 \mathrm{mM}$ aminohexanoic acid, $12 \%$ glycerol, $1 \mathrm{mM}$ PMSF, $1 \times$ complete, $2 \%$ digitonin) for 10 minutes on ice. After a clarifying spin, lysates were separated on a native $3-12 \%$ Bis-Tris gel (NativePAGE, ThermoFisher). Supercomplexes were visualized by coomassie staining or complex IV activity assay. Briefly, complex IV activi- ty was visualized by adding $2.5 \mathrm{mM} \mathrm{3,3'-Diaminobenzidine}$ dissolved in $0.05 \mathrm{M}$ phosphate buffer $\mathrm{pH} 7.4,1 \mathrm{nM}$ catalase, 1 $\mathrm{mg} / \mathrm{ml}$ cytochrome $c$ and $240 \mathrm{mM}$ sucrose.

\section{Fluorescence microscopy}

Cells were grown at $30^{\circ} \mathrm{C}$ and live images were taken using a Zeiss LSM 800 Airyscan microscope (Carl Zeiss, Jena, Germany) with a Plan-apochromatic 63X/1.4-numerical aperture oil immersion lens. For confocal excitation of GFP, a 488-nm diode laser was set at $10.0 \%$, and emission was detected between wavelengths 492 and $540 \mathrm{~nm}$. For excitation of mCherry, a $561-\mathrm{nm}$ laser line was used at $2.8 \%$, and emission was detected between 565 and $695 \mathrm{~nm}$. A Z-stack of cells was performed using ZEN blue 2.1 software and maximum intensity projections of the images are shown. Image analysis was done by using ImageJ (National Institutes of Health, Bethesda, MD) and Imaris 8.4 software.

\section{Flow cytometry}

$1 \times 10^{6}$ cells were harvested via centrifugation. Cells were washed once with water and resuspended in PBS (PBS, $25 \mathrm{mM}$ 

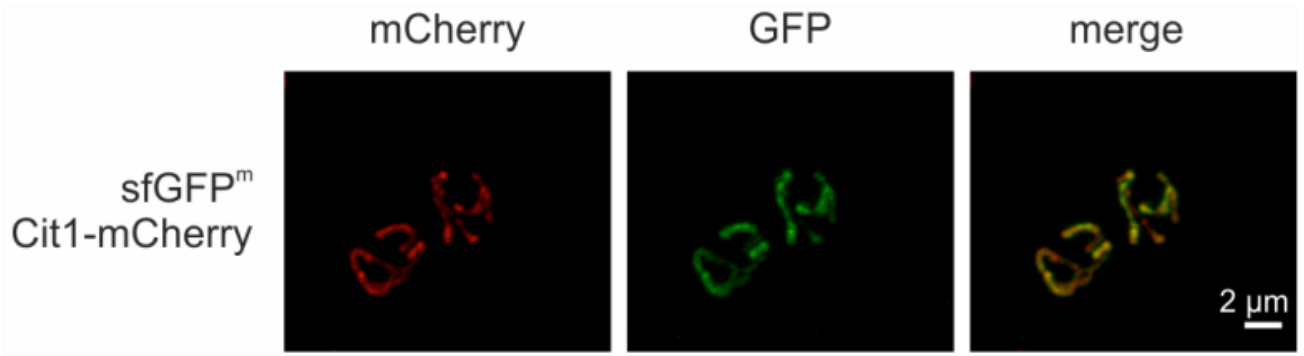

FIGURE 5: sfGFP $^{\mathrm{m}}$ visualized by fluorescence microscopy. Cells were grown to exponential phase in nonfermentable medium and sfGFP $^{m}$ and Cit1-mCherry were visualized via fluorescence microscopy.

potassium phosphate; $0.9 \% \mathrm{NaCl}$; adjusted to $\mathrm{pH} 7.2$ ). For quantification of fluorescence intensities using flow cytometry (BD LSR Fortessa), 30,000 cells were recorded and analyzed with the BD FACSDiva software. A W303a strain carrying no fluorescent tag served as background, and respective fluorescence intensities on either YPD, YPGal or YPGly were subtracted as background.

\section{Statistics}

Statistical significance was determined using student's t-test (unpaired, two-tailed, equal variance).

\section{ACKNOWLEDGEMENTS}

We thank Dr. Tom Fox (Cornell University, USA) and Dr. Alexander Tzagoloff (Columbia University, USA) for advice and material, and the members of our group for stimulating discussions. This work was supported by grants from the Knut and Alice Wallenberg Foundation, the Carl Tryggers Foundation and the Swedish Research Council. MR holds a Marie-Curie trainee grant from the horizon 2020 project remix. Part of this work was supported by the Austrian Science Fund FWF (P27183-B24) to SB.

\section{REFERENCES}

1. Borst P, Grivell LA (1978). The mitochondrial genome of yeast. Cell 15:705-723. PMID: 153200

2. Ott $M$, Amunts A, Brown A (2016). Organization and Regulation of Mitochondrial Protein Synthesis. Annu Rev Biochem 85:77-101. doi: 10.1146/annurev-biochem-060815-014334

3. Fox TD, Folley LS, Mulero JJ, McMullin TW, Thorsness PE, Hedin LO, Costanzo MC (1991). Analysis and manipulation of yeast mitochondrial genes. Methods Enzymol 194: 149-165. PMID: 1706458

4. Beugnet A, Tee AR, Taylor PM, Proud CG (2003). Regulation of targets of mTOR (mammalian target of rapamycin) signalling by intracellular amino acid availability. Biochem J 372(Pt 2): 555-566. doi: 10.1042/BJ20021266

5. Binda M, Peli-Gulli MP, Bonfils G, Panchaud N, Urban J, Sturgill TW, Loewith R, De Virgilio C (2009). The Vam6 GEF controls TORC1 by activating the EGO complex. Mol Cell 35(5): 563-573. doi: 10.1016/j.molcel.2009.06.033

6. Urban J, Soulard A, Huber A, Lippman S, Mukhopadhyay D, Deloche O, Wanke V, Anrather D, Ammerer G, Riezman H, Broach JR, De Virgilio C, Hall MN, Loewith R (2007). Sch9 is a major target of TORC1 in Saccharomyces cerevisiae. Mol Cell 26(5): 663-674. doi: 10.1016/j.molcel.2007.04.020

7. Loewith $\mathrm{R}$, Hall MN (2011). Target of rapamycin (TOR) in nutrient signaling and growth control. Genetics 189(4): 1177-1201. doi: 10.1534/genetics.111.133363

\section{SUPPLEMENTAL MATERIAL}

All supplemental data for this article are available online at www.microbialcell.com.

\section{CONFLICT OF INTEREST}

The authors declare no conflict of interest.

\section{COPYRIGHT}

(C) 2018 Suhm et al. This is an open-access article released under the terms of the Creative Commons Attribution (CC BY) license, which allows the unrestricted use, distribution, and reproduction in any medium, provided the original author and source are acknowledged.

Please cite this article as: Tamara Suhm, Lukas Habernig, Magdalena Rzepka, Jayasankar Mohanakrishnan Kaimal, Claes Andréasson, Sabrina Büttner and Martin Ott (2018). A novel system to monitor mitochondrial translation in yeast. Microbial Cell 5(3): 158-164. doi: 10.15698/mic2018.03.621

8. Wullschleger $\mathrm{S}$, Loewith $\mathrm{R}$, Hall MN (2006). TOR signaling in growth and metabolism. Cell 124(3): 471-484. doi: 10.1016/j.cell.2006.01.016

9. Suhm T, Ott M (2017). Mitochondrial translation and cellular stress response. Cell and tissue research 367(1): 21-31. doi: 10.1007/s00441-016-2460-4

10. Dai CL, Shi J, Chen Y, Iqbal K, Liu F, Gong CX (2013). Inhibition of protein synthesis alters protein degradation through activation of protein kinase B (AKT). J Biol Chem 288(33): 23875-23883. doi: 10.1074/jbc.M112.445148

11. Couvillion MT, Soto IC, Shipkovenska G, Churchman LS (2016). Synchronized mitochondrial and cytosolic translation programs. Nature 533(7604): 499-503. doi: 10.1038/nature18015

12. Cohen JS, Fox TD (2001). Expression of green fluorescent protein from a recoded gene inserted into Saccharomyces cerevisiae mitochondrial DNA. Mitochondrion 1(2): 181-189. PMID: 16120277

13. Demlow CM, Fox TD (2003). Activity of mitochondrially synthesized reporter proteins is lower than that of imported proteins and is increased by lowering cAMP in glucose-grown Saccharomyces cerevisiae cells. Genetics 165(3): 961-974. PMID:14668357.

14. Pedelacq JD, Cabantous S, Tran T, Terwilliger TC, Waldo GS (2006). Engineering and characterization of a superfolder green fluorescent protein. Nat Biotechnol 24(1): 79-88. doi: 10.1038/nbt1172 
15. Gruschke S, Römpler K, Hildenbeutel M, Kehrein K, Kühl I, Bonnefoy N, Ott M (2012). The Cbp3-Cbp6 complex coordinates cytochrome $b$ synthesis with $b c_{1}$ complex assembly in yeast mitochondria. J Cell Biol 199(1): 137-150. doi: 10.1083/jcb.201206040

16. Ding MG, Butler CA, Saracco SA, Fox TD, Godard F, di Rago JP, Trumpower BL (2008). Introduction of cytochrome $b$ mutations in Saccharomyces cerevisiae by a method that allows selection for both functional and non-functional cytochrome b proteins. Biochim Biophys Acta 1777(9): 1147-1156. doi: 10.1016/j.bbabio.2008.04.029

17. Perez-Martinez X, Broadley SA, Fox TD (2003). Mss51p promotes mitochondrial Cox1p synthesis and interacts with newly synthesized Cox1p. EMBO J 22(21): 5951-5961. doi: 10.1093/emboj/cdg566

18. Costanzo MC, Fox TD (1990). Control of mitochondrial gene expression in Saccharomyces cerevisiae. Annu Rev Genet 24:91-113. doi: 10.1146/annurev.ge.24.120190.000515

19. Bonnefoy N, Fox TD (2007). Directed alteration of Saccharomyces cerevisiae mitochondrial DNA by biolistic transformation and homologous recombination. Methods Mol Biol 372:153-166. doi: 10.1007/978-1-59745-365-3_11

20. Bonnefoy N, Fox TD (2000). In vivo analysis of mutated initiation codons in the mitochondrial COX2 gene of Saccharomyces cerevisiae fused to the reporter gene $A R G 8^{m}$ reveals lack of downstream reinitiation. Mol Gen Genet 262(6): 1036-1046. PMID: 10660064

21. Rosenkrantz M, Alam T, Kim KS, Clark BJ, Srere PA, Guarente LP (1986). Mitochondrial and nonmitochondrial citrate synthases in Saccharomyces cerevisiae are encoded by distinct homologous genes. Mol Cell Biol 6(12): 4509-4515. PMID: 3540614.
22. Trumbly RJ (1992). Glucose repression in the yeast Saccharomyces cerevisiae. Mol Microbiol 6(1): 15-21. PMID: 1310793

23. Carlson M (1999). Glucose repression in yeast. Curr Opin Microbiol 2(2): 202-207. doi: 10.1016/S1369-5274(99)80035-6

24. Ronne H (1995). Glucose repression in fungi. Trends Genet 11(1): 12-17. PMID: 7900189

25. Thorsness PE, Fox TD (1993). Nuclear mutations in Saccharomyces cerevisiae that affect the escape of DNA from mitochondria to the nucleus. Genetics 134(1): 21-28. PMID: 8514129

26. Fox TD (1979). Five TGA "stop" codons occur within the translated sequence of the yeast mitochondrial gene for cytochrome $c$ oxidase subunit II. Proc Natl Acad Sci U S A 76(12): 6534-6538. PMID: 230513.

27. Steele DF, Butler CA, Fox TD (1996). Expression of a recoded nuclear gene inserted into yeast mitochondrial DNA is limited by mRNAspecific translational activation. Proc Natl Acad Sci USA 93(11): 52535257. PMID: 8643562

28. Dunstan HM, Green-Willms NS, Fox TD (1997). In vivo analysis of Saccharomyces cerevisiae COX2 mRNA 5'-untranslated leader functions in mitochondrial translation initiation and translational activation. Genetics 147(1): 87-100. PMID: 9286670

29. Silve S, Volland C, Garnier C, Jund R, Chevallier MR, HaguenauerTsapis $R$ (1991). Membrane insertion of uracil permease, a polytopic yeast plasma membrane protein. Mol Cell Biol 11(2): 1114-1124. PMID: 1846664 